\title{
Augmentation of conductive pathways in carbon black/PLA 3D-printed electrodes achieved through
}

varying printing parameters

A. Abdalla ${ }^{1,2}$, H.H. Hamzah ${ }^{1}$, O. Keattch ${ }^{3}$, D. Covill ${ }^{3,4}$, B.A. Patel ${ }^{1,2^{*}}$

${ }^{1}$ School of Pharmacy and Biomolecular Sciences, ${ }^{2}$ Centre for Stress and Age-Related Disease ${ }^{3}$ School of Computing, Engineering and Mathematics and ${ }^{4}$ Centre for Regenerative Medicine and Devices, Brighton, UK, BN2 4GJ.

${ }^{*}$ Correspondence should be addressed to Prof. Bhavik Patel; Email: b.a.patel@brighton.ac.uk; Tel: +44(0)1273 642418 


\section{ABSTRACT}

3D-printing of conductive carbon materials in sensing applications and energy storage devices has significant potential, however high resistivity of 3D-printed filaments poses a challenge. Strategies to enhance sensors post printing are time consuming and can reduce structural integrity. In this work, we investigated the effects different printing layer thickness and orientation can have on the electron transfer kinetics and resistivity of conductive materials. The response of these electrodes was investigated by cyclic voltammetry, electrochemical impedance spectroscopy and imaging. Electrodes printed with the lowest layer thickness of $0.1 \mathrm{~mm}$ in a vertical orientation had the greatest conductivity. With increasing print layer thickness and printing in a horizontal orientation, the electrode was more resistive. This work is the first to demonstrate the significant impact 3D-printing parameters can have on the electron transfer kinetics of carbon conductive electrodes. The implications of this study are important in defining the manufacturing process of electrodes for all applications.

Keywords: 3D-printing, additive manufacturing, conductive pathways, electron transfer, sensors 


\section{Introduction}

Three-dimensional (3D) fused filament fabrication (FFF) printing has emerged as an important manufacturing approach for the development of conductive carbon materials, mainly in the fields of electronics, sensors and energy storage devices as it offers flexibility in design and potential that supersedes traditional manufacturing systems. [1-6] Studies have shown that commercial conductive carbon 3D-printed filament has poor conductivity. [7-10] To overcome these limitations, varying postprinting modifications have been utilised in order to enhance the electrical properties of the 3D-printed material, such as chemical and electrochemical treatment. [11-14] Although these strategies can effectively enhance the electron transfer kinetics of the printed electrode, [15] they add an additional time consuming step, which is often detrimental to the electrode geometry when making small fine prints.

On the other hand, studies that focus on enhancing the conductivity of 3D-printed devices through the improvement of printing parameters are seldom explored. [16-18] There are various parameters that govern the properties of the printed artefact. Studies have shown that anisotropy and orientation of print layers are two factors in the construct of 3D-printed materials which introduce significant variations in electrochemical activity. [18] This study aims to understand the influence of print layer thickness and orientation on the electron transfer kinetics of 3D-printed carbon black (CB)/polylactic acid (PLA) electrodes

Herein, we utilise cone-shaped CB/PLA electrodes printed with layer thicknesses varying from 0.1 to $0.4 \mathrm{~mm}$ in vertical and horizontal orientation. Electrochemical characterisation was conducted using voltammetric measurements of two different redox species, via cyclic voltammetry (CV) and impedance electrochemical 
spectroscopy (EIS). Structural integrity of the different layer thicknesses was analysed through chemical pre-treatment with tetrahydrofuran (THF) while the CB particles present within the 3D-printing filament were characterized using SEM and particle size analysis. Our findings show that layer thickness and printing orientation has significant influence on the electrochemical activity of the printed material and determines the prevalence of conductive pathways and resistivity within the structure.

\section{Experimental}

\subsection{Fabrication of 3D-printed carbon black/PLA electrodes of varying layer thickness}

For the electrodes, a cone with a $4.5 \mathrm{~mm}$ diameter at base and length of $3 \mathrm{~mm}$ was printed using a commercial polylactic acid (PLA) / carbon black (CB) filament (Protopasta, WA, USA) at varying layer thicknesses in either a horizontal or vertical orientation (Figure 1). The electrodes were printed on a Wanhao Duplicator 4 with a $0.4 \mathrm{~mm}$ brass nozzle at $220^{\circ} \mathrm{C}$ and a bed temperature of $50{ }^{\circ} \mathrm{C}$ at $3600 \mathrm{~mm} / \mathrm{min}$. The layer height was varied from 0.1 to $0.4 \mathrm{~mm}$, with 2 shells (outer perimeter toolpaths) and $100 \%$ infill density. Two skirt outlines were used to prime the extruder. To compensate for the filler content of the material, retractions were disabled, and the extrusion multiplier was set to 1.1. The cone electrodes were placed on top of an acrylic tube and sealed with Araldite. Connection was achieved by attaching a copper wire to the electrode using CircuitWorks Conductive (Silver) epoxy. 


\subsection{Electrochemical characterisation of 3D-printed electrodes}

Electrochemical assessments on the 3D-printed cone electrodes were carried out using a three-electrode system, which consisted of a $\mathrm{Ag} \mid \mathrm{AgCl}(3 \mathrm{M} \mathrm{KCl})$ reference electrode, a platinum wire auxiliary electrode and the 3D-printed electrode as the working electrode. All electrochemical experiments were carried out using a $\mathrm{CH}$ instrument potentiostat/galvanostat $\mathrm{CHI} 760 \mathrm{E}(\mathrm{CH}$ instruments, Texas). The electrochemical characteristics of the 3D-printed electrodes were assessed using the redox couples of $1 \mathrm{mM}$ hexaammineruthenium(III) chloride in $1 \mathrm{M} \mathrm{KCl}$ and $1 \mathrm{mM}$ serotonin in Krebs' buffer solution, pH $7.4(117 \mathrm{mM} \mathrm{NaCl}, 4.7 \mathrm{mM} \mathrm{KCl}, 2.5 \mathrm{mM} \mathrm{CaCl}$, $1.2 \mathrm{mM} \mathrm{MgCl}_{2}, 1.2 \mathrm{mM} \mathrm{NaH}_{2} \mathrm{PO}_{4}, 25 \mathrm{mM} \mathrm{NaHCO}_{3}$ and $11 \mathrm{mM}$ glucose). Cyclic voltammograms were performed with a scan rate of $100 \mathrm{mV} / \mathrm{s}$. Electrochemical Impedance Spectroscopy (EIS) measurements were performed in a mix of $0.5 \mathrm{mM}$ potassium ferricyanide and $0.5 \mathrm{mM}$ potassium ferrocyanide in $1 \mathrm{M} \mathrm{KCl}$ at a potential equal to the anodic potential. A frequency range of $100 \mathrm{kHz}$ to $0.01 \mathrm{~Hz}$ and an amplitude of $5 \mathrm{mV}$ were utilised.

\subsection{Percentage weight and particle size of $C B$ in filament}

To determine the mass composition of the CB present in the CB/PLA filament, samples of the filament were weighed and placed in $20 \mathrm{~mL}$ Tetrahydrofuran (THF). The mixture was then sonicated for 5 minutes followed by vacuum filtration. Samples were left overnight to dry and rewashed with THF to ensure all PLA is removed from sample. The dry sample was then reweighed to calculate the percent composition of CB. To analyze the particle sizes, present in these samples, the dried samples were washed a few times in deionized water, followed by sonication in $10 \mathrm{ml}$ of the same to ensure 
adequate dispersion. The wet mixture was then run in a Malvern Instruments Mastersizer (Malvern, UK) with a particle refractive index of 1.416, absorption index of 1.0 and dispersant refractive index of 1.33

\subsection{Scanning electron microscopy (SEM)}

The samples were imaged using a Zeiss SIGMA field emission gun SEM equipped with an Everhart-Thornley detector operating in secondary electron detection mode, using $5 \mathrm{kV}$ accelerating voltage, a $20 \mu \mathrm{m}$ aperture, and $8.1 \mathrm{~mm}$ working distance. Samples tested were powdered CB prepared as explained above in sample treatment section, and single layer of CB/PLA printed at layer thickness of $0.2 \mathrm{~mm}$, which was placed in THF for 30 seconds to erode surface PLA to showcase the presence of CB aggregates within the filament structure.

\subsection{Microscopy}

Single layers of CB/PLA printed at layer thickness of $0.1 \mathrm{~mm}$ to $0.4 \mathrm{~mm}$ were printed on a Creality Ender Pro 3, with a $0.4 \mathrm{~mm}$ steel nozzle at $230^{\circ} \mathrm{C}$ and a bed temperature of $90{ }^{\circ} \mathrm{C}$ at $3600 \mathrm{~mm} / \mathrm{min}$. Two shells (outer perimeter toolpaths) and $100 \%$ infill density were used and 2 skirt outlines were used to prime the extruder. To compensate for the filler content of the material, retractions were disabled, and the extrusion multiplier was set to 1.1. These single layers were placed in THF between $10-30$ seconds $(0.1 \mathrm{~mm})$ and 3-5 minutes $(0.4 \mathrm{~mm})$ until structural deformities could be seen under a light microscope. Subsequently, they were imaged to further analyze the structural deformities formed. Images of vertical and horizontal printed sensors at all different 
thicknesses were also obtained and apparent surface area was measured on ImageJ to determine differences between orientations and print thicknesses.

\subsection{4-point probe measurement of conductivity}

4-point probe system (Ossila Ltd, UK) was utilized for all resistivity measurements. 3D-printed sensors were each measured at center of sample (sensor replicates $=4$ 5) at different orientations and thicknesses. CB/PLA filament at 10 * $1.75 \mathrm{~mm}$ (replicates $=4$ ) were also tested with probes placed on the center of cylinder axis. Sheet resistance was measured using script with current at $1 \mu \mathrm{A}$. Resistivity was calculated as the follows: [19]

$$
\begin{gathered}
\text { Resistivity }=2 * \pi * \mathrm{~s} * \frac{\Delta \mathrm{V}}{\mathrm{I}} * F_{\text {thickness }} * F_{\text {diameter }} \\
F_{\text {diameter }}=\frac{\ln 2}{\ln 2+\ln \left(\frac{d^{2}}{s^{2}}+3\right)-\ln \left(\frac{d^{2}}{s^{2}}-3\right)}
\end{gathered}
$$

Where resistivity is measured in $\Omega . \mathrm{cm}, \mathrm{s}$ is probe spacing $(0.1 \mathrm{~cm}), \Delta \mathrm{V}$ potential change recorded by 4 -pont probe, I is current sourced $(1 \mu \mathrm{A}), \mathrm{F}_{\text {thickness }}$ is thickness correction factor from table based on length and width (CB/PLA filament or sensors), Fdiameter is correction factor for diameter, $\mathrm{d}$ is diameter $(0.4 \mathrm{~cm}$ for sensors and $0.175 \mathrm{~mm}$ for filament). [19]

\subsection{Data analysis}

The cyclic voltammetry measurements were analysed for anodic/cathodic peak potential and current, as well as the difference in the anodic/cathodic peak potential $(\triangle \mathrm{E})$ obtained from the voltammograms using $\mathrm{CHI} 760 \mathrm{E}$ software. The heterogenous 
electron transfer kinetics (HET, $k^{\circ}$ ) were calculated based on the method by Nicholson as follows.[20]

$$
k^{\mathrm{o}}=\Psi \sqrt{\left(\pi * \frac{n F}{R T} * D * \mathrm{v}\right)}
$$

Where $\Psi$ is dimensionless charge transfer parameter as tabulated by Nicholson [20], $\mathrm{R}$ is gas constant $\left(\mathrm{J} \mathrm{K}^{-1} \mathrm{~mol}^{-1}\right), \mathrm{T}$ is temperature $(\mathrm{K}), \mathrm{F}$ is Faraday constant, $\mathrm{n}$ is a number of electrons transfer, $D$ is diffusion coefficient $\left(\mathrm{cm}^{2} / \mathrm{s}\right)$, and $\mathrm{v}$ is scan rate $(\mathrm{V} / \mathrm{s})$. Microscopy images were analysed with ImageJ where surface area was calculated pre and post treatment in THF for all layer thicknesses. For all measurements, the data was presented as mean and its associated standard deviation and statistically compared using one-way ANOVA test and two-way ANOVA, with post hoc tests where appropriate.

\section{Results and discussion}

The enhancement of electrochemical activity thought manipulation of 3D-printng parameters has rarely been studied in comparison to methods such as electrochemical pre-treatment or chemical pre-treatment which are post-printing methods. Herein, we study the effects on printing CB/PLA sensors vertically versus horizontally, at different layer thicknesses ranging from $0.1-0.4 \mathrm{~mm}$.

Figure 1 shows the cone shaped electrodes printed in varying layer thicknesses and orientation. The geometric surface area of electrodes did not significantly vary with respect to layer thickness and orientation (Figure S1). In the vertical orientation, the individual print layers were aligned in the direction of electrical connection and solution 
interface, whilst in the horizontal orientation, the print layers were perpendicular to the electrical connection and solution interface. Thus, it is important to note here, that for vertical electrodes, each printed layer is aligned from the electrical connection to the solution interface such as a forest of layers, whereas the horizontal electrodes are a stack of printed layers, in which connection must be found between each layer in order to have successful conductive pathway from electrical connection to the solution interface.

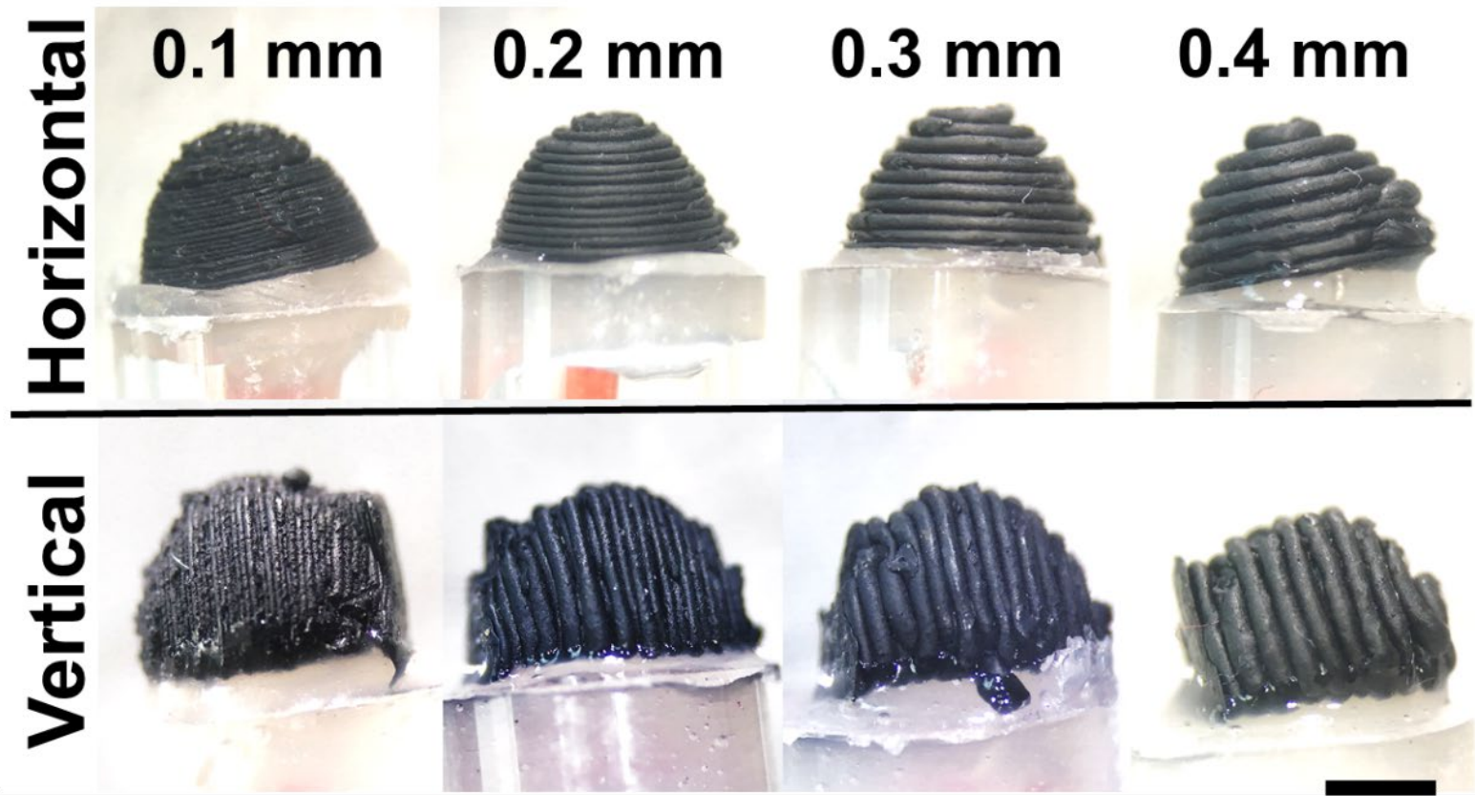

Figure 1. Optical microscope side images of 3D-printed CB/PLA electrodes printed in horizontal and vertical orientations at layer thicknesses between 0.1 to $0.4 \mathrm{~mm}$. Electrical connection is vertically below the electrodes. Scale bar is $2 \mathrm{~mm}$.

\subsection{Electrochemical activity varies with orientation and layer thickness for outer and inner-sphere redox molecules}

Electrochemical characterisation of these electrodes is shown in Figure 2 for outer sphere redox species hexaamineruthenium (III) chloride. [21] In the vertical orientation 
print, clear voltammetric peaks were observed (Figure $2 A, n=4$ ), with significant reduction in the cathodic peak current $(\mathrm{Ipc})$ in $0.4 \mathrm{~mm}$ print layer thickness when compared to 0.1 and $0.2 \mathrm{~mm}$ (Figure $2 \mathrm{~B}, \mathrm{p}<0.05, \mathrm{n}=4$ ). In addition, there was a significant increase in peak to peak separation $(\Delta E)$ in $0.3 \mathrm{~mm}(p<0.01)$ and $0.4 \mathrm{~mm}$ $(p<0.001)$ when compared to $0.1 \mathrm{~mm}$ layer thickness (Figure $2 \mathrm{C}, \mathrm{n}=4$ ). Additionally, a significant increase in $\Delta \mathrm{E}$ in $0.3 \mathrm{~mm}$ and $0.4 \mathrm{~mm}$ layer thickness was observed when compared to $0.2 \mathrm{~mm}(p<0.001)$. Finally, the $\Delta E$ was significantly greater in $0.4 \mathrm{~mm}$ when compared to $0.3 \mathrm{~mm}$ print layer thickness (Figure $2 \mathrm{C}, \mathrm{p}<0.01, \mathrm{n}=4$ ). Voltammograms for horizontally printed electrodes displayed more peak to peak separation (Figure 2D, n=4). No significant difference in the Ipc (Figure 2E, $n=4$ ) and $\Delta \mathrm{E}$ (Figure $2 \mathrm{~F}, \mathrm{n}=4$ ) was observed between different layer thickness of horizontally printed electrodes. However, $\Delta \mathrm{E}$ was smaller in electrodes printed vertically at layer thicknesses between 0.1 to $0.3 \mathrm{~mm}$ when compared to horizontal printed electrodes $(p<0.01)$
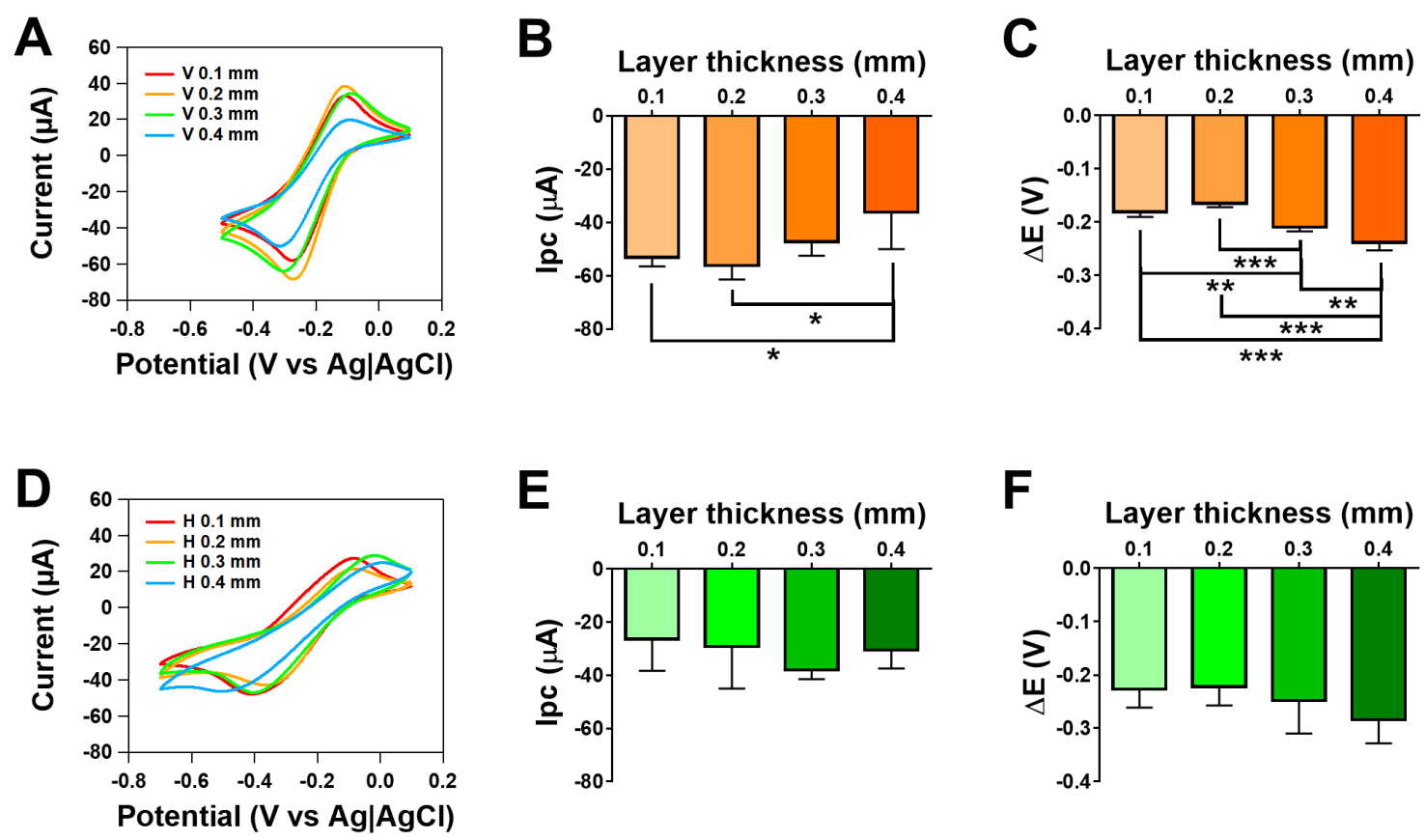
Figure 2. Voltammetric responses of hexaamineruthenium (III) chloride. (A) Cyclic voltammograms in vertical electrodes, where the (B) Ipc and (C) $\Delta E$ from multiple electrodes of varying layer thickness are shown. (D) Cyclic voltammograms in horizontal electrodes, where the $(E)$ Ipc and $(F) \Delta E$ from multiple electrodes of varying layer thickness are shown. Data shown as mean \pm standard deviation (S.D.), where $n=4,{ }^{*} p<0.05,{ }^{* *} p<0.01$ and ${ }^{* * *} p<0.001$.

Inner-sphere redox molecule, serotonin, [22] was also explored to study differences in electrochemical behaviour between prints (Figure S2). There was a significant increase in the anodic current observed on vertically printed electrodes when compared to horizontal printed electrodes $(p<0.05, n=4)$. The vertical print had a significant reduction in the anodic peak potential in $0.4 \mathrm{~mm}$ when compared to $0.1 \mathrm{~mm}$ $(p<0.01), 0.2 \mathrm{~mm}(p<0.001)$ and $0.3 \mathrm{~mm}(p<0.05, n=4$, Figure S2B $)$. For the horizontal print, a significant reduction in the anodic peak current in $0.3 \mathrm{~mm}(p<0.05)$ and $0.4 \mathrm{~mm}$ $(p<0.01)$ was seen when compared to $0.1 \mathrm{~mm}$ layer thickness $(n=4$, Figure S2E). Given the lack of peak definition, the oxidation peak potential was difficult to obtain, however there was a trend of an increasing oxidation peak potential for larger print layers in the vertical print. Hence, vertically printed sensors at layer thicknesses $0.1-$ $0.2 \mathrm{~mm}$ showed enhanced electrochemical activity for both molecules.

\subsection{Enhancement of conductivity in vertical orientation and smaller layer thickness printed electrodes}

The electron transfer rate $\left(\mathrm{k}^{\circ}\right)$, calculated by the Nicholson method, $[20,23,24]$ display a trend of faster electron transfer kinetics for vertically printed electrodes at print layer thickness of $0.1-0.2 \mathrm{~mm}$, with the $0.4 \mathrm{~mm}$ vertical electrodes showing similar kinetics 
to the horizontal printed electrodes of $0.1-0.2 \mathrm{~mm}$ (Figure $3 \mathrm{~A}$ ). Furthermore, there was a significant decrease in $\mathrm{k}^{\circ}$ between vertical and horizontal electrodes for all printed orientations except $0.4 \mathrm{~mm}(\mathrm{p}<0.001, \mathrm{n}=4)$. There was also a significant decrease in the $k^{\circ}$ in both horizontal and vertical electrodes with increasing print layer thickness, where vertically $0.2 \mathrm{~mm}$ print $(p<0.01)$ had a significantly higher $\mathrm{k}^{\circ}$ than $0.1 \mathrm{~mm}$ but $0.3-0.4 \mathrm{~mm}$ prints had a significantly lower $\mathrm{k}^{\circ}$ than $0.1-0.2 \mathrm{~mm}$ prints $(\mathrm{p}<0.001)$. The $0.4 \mathrm{~mm}$ print was significantly lower than $0.3 \mathrm{~mm}(p<0.01)$. Horizontally, no significant difference was seen in the electron transfer kinetics. The $k^{0}$ was smaller in lower print thickness than in higher print thicknesses or horizontally printed electrodes, which indicate more conductive pathways and hence faster electron kinetics in vertical versus horizontal orientation at smaller prints.

Using 4-point probe measurement for resistivity (Figure 3B), the $0.1 \mathrm{~mm}(p<0.01, n=4)$, $0.2 \mathrm{~mm}$ and $0.4 \mathrm{~mm}$ prints $(0.001, \mathrm{n}=5)$ are all less resistive in the vertical prints when compared to horizontal prints. Vertically, increasing layer thickness also displayed a similar trend where $0.3 \mathrm{~mm}(p<0.05, n=5)$, and $0.4 \mathrm{~mm}$ prints $(p<0.001, n=5)$ were more resistive than $0.1-0.2 \mathrm{~mm}$ prints. Horizontally, $0.1 \mathrm{~mm}$ print was less resistive than $0.2 \mathrm{~mm}(\mathrm{p}<0.01, \mathrm{n}=4)$ and $0.4 \mathrm{~mm}$ prints $(\mathrm{p}<0.001, \mathrm{n}=5)$, whilst the $0.3 \mathrm{~mm}$ print was less resistive than both 0.2 and $0.4 \mathrm{~mm}$ prints $(p<0.001, n=4)$.

For semi-infinite 3D-bulk material such as our sensors, a 4-point probe sources current spherically from the middle probes. For larger print thicknesses, the larger resistivity found in both vertical and horizontal prints could denote a less structured conductive pathway that the current can flow through, with PLA between the CB particles possibly increasing resistivity. Similarly, the horizontal prints, display higher resistivity than their vertical counterparts, suggestive of poor distribution of conductive materials within composite. [25] As the filament is extruded, the CB particles are compacted and more 
ordered in thinner layers, creating a greater probability for additional conductive routes in vertical prints. As the layer thickness increases, the position of the CB particles can be less ordered within a single print layer, thus resulting in reduced conductive pathways. Horizontally, conductive pathways need to occur between each print layer and therefore point-to-point conductive contact between print layers seems more likely in $0.1 \mathrm{~mm}$ print layer thickness where $\mathrm{CB}$ particles are more ordered due to geometric constraints. Furthermore, this high resistivity is also likely to be a result of the voids that form between printed layers during the FFF printing process, which has been shown to increase in number at larger layer thicknesses. [26-30] These air-filled voids decrease the surface area of point-to-point contact necessary for the formation of successful conductive pathways thus increasing the resistivity at higher prints and in horizontal orientation of print.

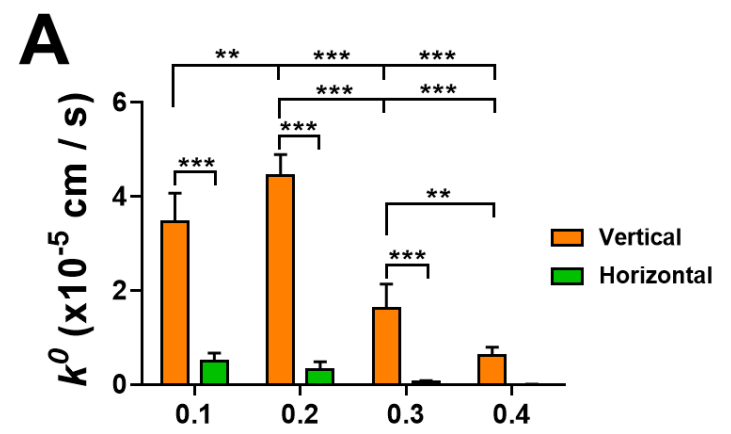

Layer thickness $(\mathrm{mm})$

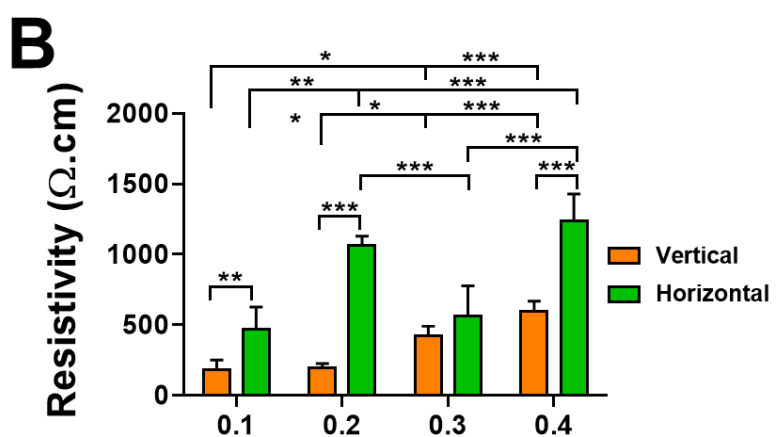

Layer thickness $(\mathrm{mm})$

Figure 3. (A) Mean heterogeneous electron transfer $\left(k^{\circ}\right)$ constants from the electrodes printed at varying layer thickness in either vertical or horizontal orientation $(n=4)$. (B) 4-point resistivity measurements for sensors printed at varying layer thickness in either vertical or horizontal orientation ( $n=4-5$. Data shown as mean \pm standard deviation (S.D.), ${ }^{*} p<0.05$, ${ }^{* *} p<0.01$ and ${ }^{* * *} p<0.001$. 
Electrochemical impedance was utilised to understand the internalised structure of the printed electrode using a potassium ferric/ferrocyanide mixture as is standard for these measurements.[31-33] From the Nyquist plots for electrodes printed at varying layer thicknesses in vertical (figure 4A) and horizontal (figure 4D) orientation, the interfacial charge-transfer resistance $\left(R_{\mathrm{ct}}\right)$ and uncompensated solution resistance $\left(R_{\mathrm{s}}\right)$ was obtained. There was a significantly decreased $R_{\mathrm{ct}}$ in vertically printed electrodes when compared to the horizontal $(p<0.01, n=4)$, which supports our previously published findings, which observed reduced $R_{\mathrm{ct}}$ in the vertical direction of single print layers in comparison to the horizontal direction of 3D-printed CB/ABS electrodes. [18] Figure $4 \mathrm{~B}$ shows that there is a significantly larger $R_{\mathrm{ct}}$ in the vertical $0.4 \mathrm{~mm}$ print layer thickness when compared to $0.1 \mathrm{~mm}(p<0.01), 0.2 \mathrm{~mm}(p<0.001)$ and $0.3 \mathrm{~mm}(p<0.01)$ electrodes $(\mathrm{n}=4)$. There was no difference in the $R_{\mathrm{s}}$ in all vertical electrodes of varying print layer thickness. Figure $4 \mathrm{E}$ shows that there is a signficantly greater $R_{\mathrm{ct}}$ in the hortizonal $0.4 \mathrm{~mm}$ printed electrodes in comparison to the $0.1 \mathrm{~mm}$ electrodes $(p<0.05$, $\mathrm{n}=4$ ). In addition, there is a signficantly greater $R_{\mathrm{s}}$ in the horizontal $0.4 \mathrm{~mm}$ print thickness electrode when compared to $0.1 \mathrm{~mm}$ thickness electrode $(p<0.01, n=4$, figure 4F).

Results from impedance studies futher support the observed voltammetric responses and resultant values of $\mathrm{k}^{\circ}$ in indicating that 3D-printing of small layer thickneses assessed in a vertical electrode provided the greatest degree of conductive pathways. This ordered conductive stucture diminishes with increasing layer thickness and if the electrode is printed in a horizontal orientation. This difference in vertical and horiozontal prints is mainly a result of the orientation of print to the the orientation of electrical connection. In vertical electrodes, which are parallel to the electrical connection and solution interface, each print layer acts as an individual electrode, and 
the resultant electrode is like a forest of individual printed strands. The presence of voids between the vertically printed strands in such a structure will be of minimum effect, as the need for connections between the layers is not essential for establishment of conductive pathways. However, within the horizontal print, where point-to-point contact needs to be made between the printed layers to achieve conductive pathways there was a reduced conductivity with increased print layer thickness. In this instance, the presence of air voids play a significant concentrtion in causing an increase in $R_{\mathrm{ct}}$ at horizontal prints, and more so at the larger prints.
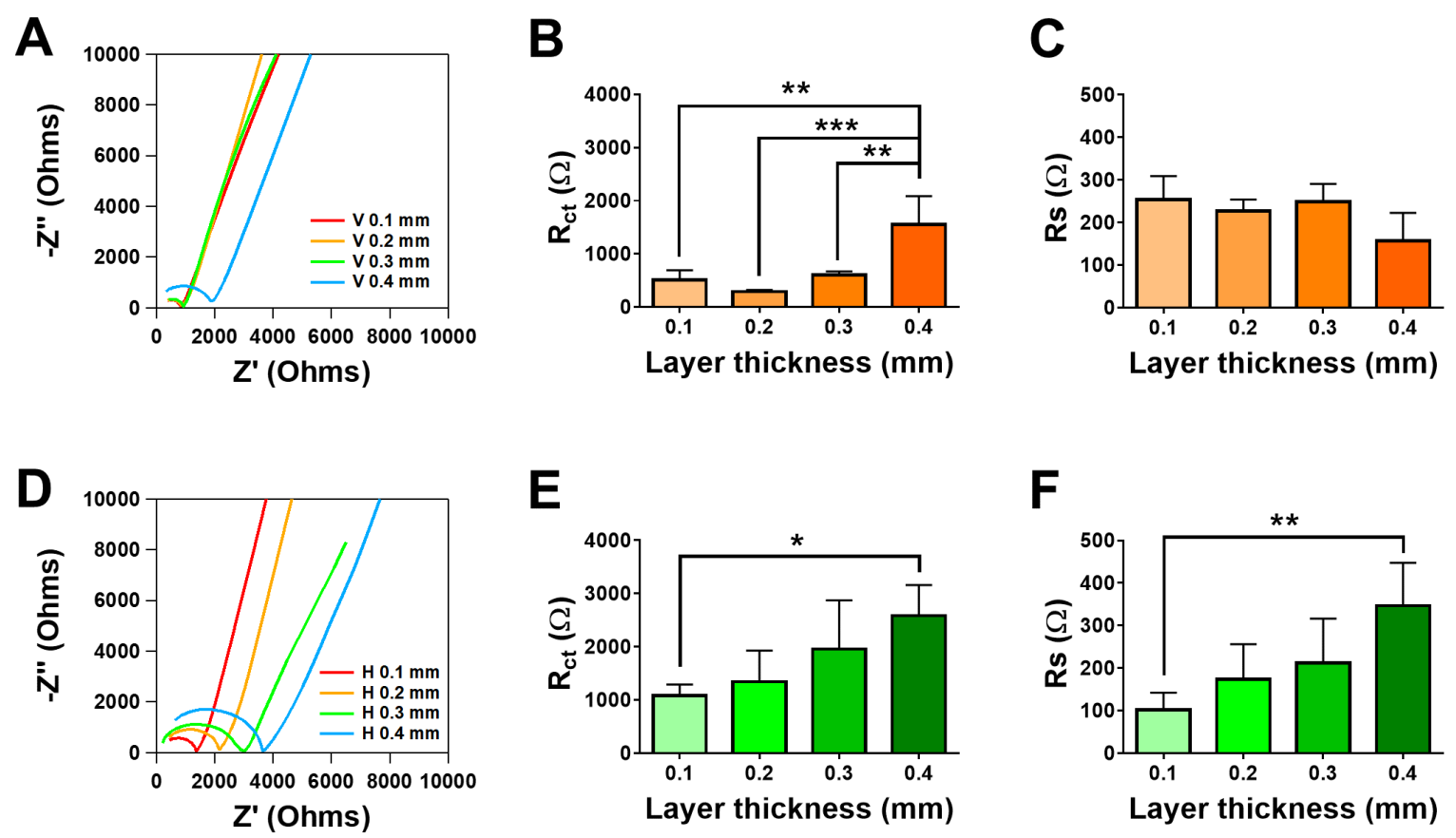

Figure 4. Determination of solution and charge transfer resistance. (A) Nyquist representations of the impedance spectra at $\sim 0.6 \mathrm{~V} v s \mathrm{Ag} \mid \mathrm{AgCl}$ in $10 \mathrm{mM}$ of $\mathrm{K}_{4}\left[\mathrm{Fe}(\mathrm{CN})_{6}\right] / \mathrm{K}_{3}\left[\mathrm{Fe}(\mathrm{CN})_{6}\right]$ in $1 \mathrm{M} \mathrm{KCl}$ for vertically printed electrodes. The measurements were made at a frequency range from $100 \mathrm{kHz}$ to $0.1 \mathrm{~Hz}$ with a modulation amplitude of $5 \mathrm{mV}$. (B) shows a comparison of the charge transfer resistance (Rct), and $(C)$ uncompensated solution resistance $(R \mathrm{~s})$ for vertically printed electrodes. (D) Nyquist representations for horizontally printed electrodes. (E) Rct and (C) Rs for horizontally printed electrodes. Data are shown as mean \pm S.D., $n=4,{ }^{*} p<0.05,{ }^{* *} p<0.01$ and ${ }^{* * *} p<0.001$. 


\subsection{Internalized structure and arrangement of CB/PLA particles determines success in formation of conductive pathways}

We investigated the composition of the CB filament in order to understand the potential structure of the 3D-printed carbon composite electrode. The outer layer of the PLA was etched off using treatment with THF, where clear aggregation of CB particles was observed (Figure 5A). These findings are similar to other studies who have explored the structure of conductive carbon 3D printable filaments. [34, 35] The conductive filament was dissolved in THF to remove PLA [36] and the resultant filtrate indicated that $53 \pm 7 \%(n=10)$ of CB by mass ( $23-30 \%$ by volume) was present in the filament. The particle size distribution of CB was subsequently studied using SEM and a Mastersizer. Figure 5B and C shows a wide distribution of particle sizes present in the filament ranging from fine particles at $5 \mu \mathrm{m}$ to aggregations around $400 \mu \mathrm{m}$. Aggregations of particles were like those observed in the filament. The particle size analysis (Figure 5D) further showed a similar distribution, where the median particle size by volume was $224 \pm 132 \mu \mathrm{m}(\mathrm{n}=8)$.

Given that half the filament by mass contained CB particles, the structuring of this conductive component was explored by removing the PLA using THF from a single print layer (where the time of treatment was normalised by the print layer mass and change in surface area was monitored by image analysis as shown in Figure S3). In the different print layer thicknesses, the amount of PLA lost during THF treatment was not significantly different (Figure $5 \mathrm{E}$ ), but significantly less than total composition of PLA. This suggest our treatment removed surface layers of PLA. The $0.4 \mathrm{~mm}$ print layer shows cracks and crevices after treatment with THF due to PLA removal, showcasing the harsh nature of chemical pre-treatment on preservation of structural geometry. The $0.1 \mathrm{~mm}$ layer seems to hold its structural integrity, where PLA seems 
to be equally eroded off the outer surface of the print (Figure 5F). The small distortion in the printed line seen in the $0.1 \mathrm{~mm}$ specimens can be attributed to the over extrusion that occurs when printing layer heights are less than half the nozzle diameter. Thus, for the $0.4 \mathrm{~mm}$ nozzle diameter used, a small distortion is expected when printing 0.1 $\mathrm{mm}$, but thus far this has not shown any drawbacks on the conductivity of the specimen used as displayed in the experiments above.
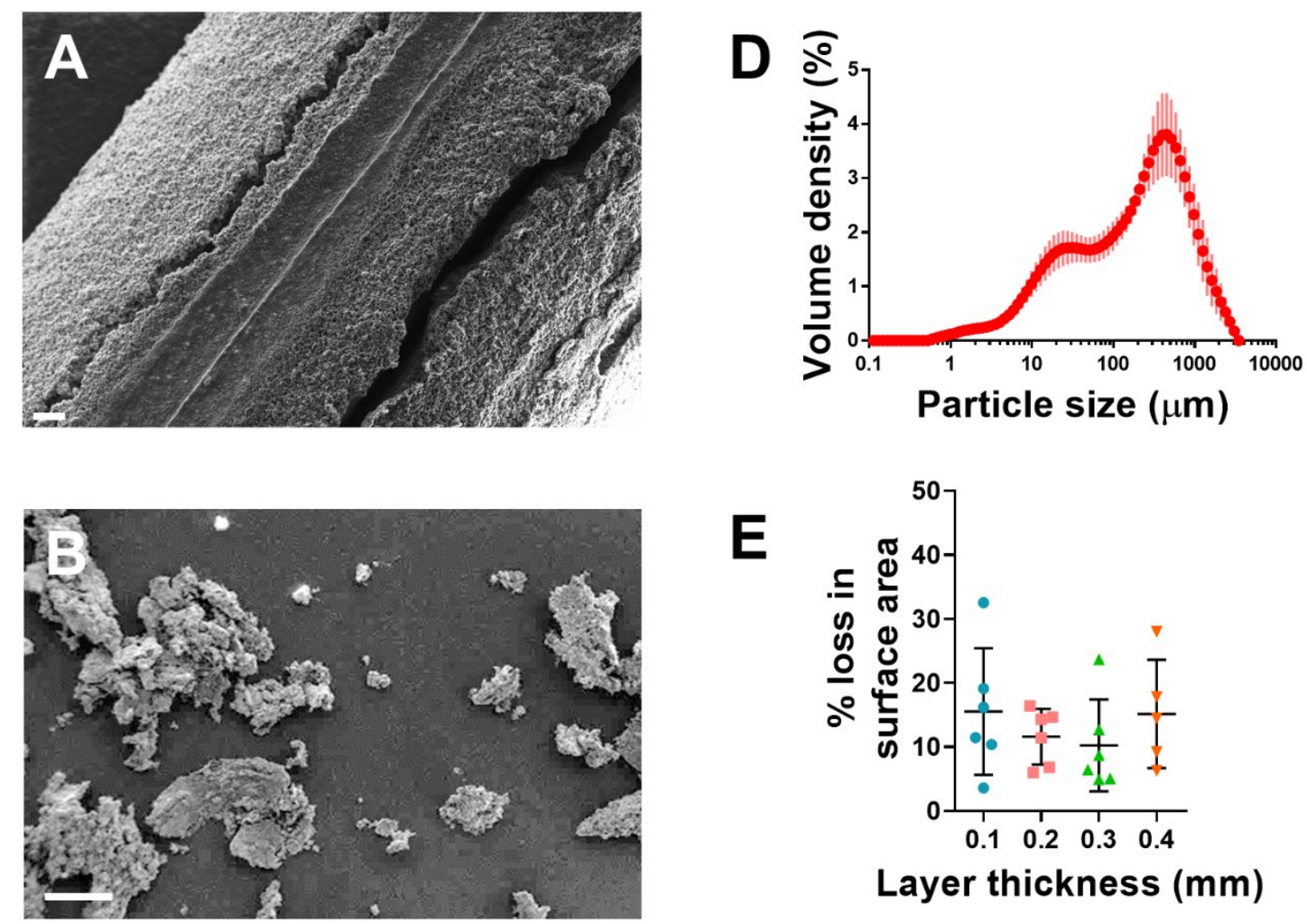

\section{Layer thickness $(\mathrm{mm})$}
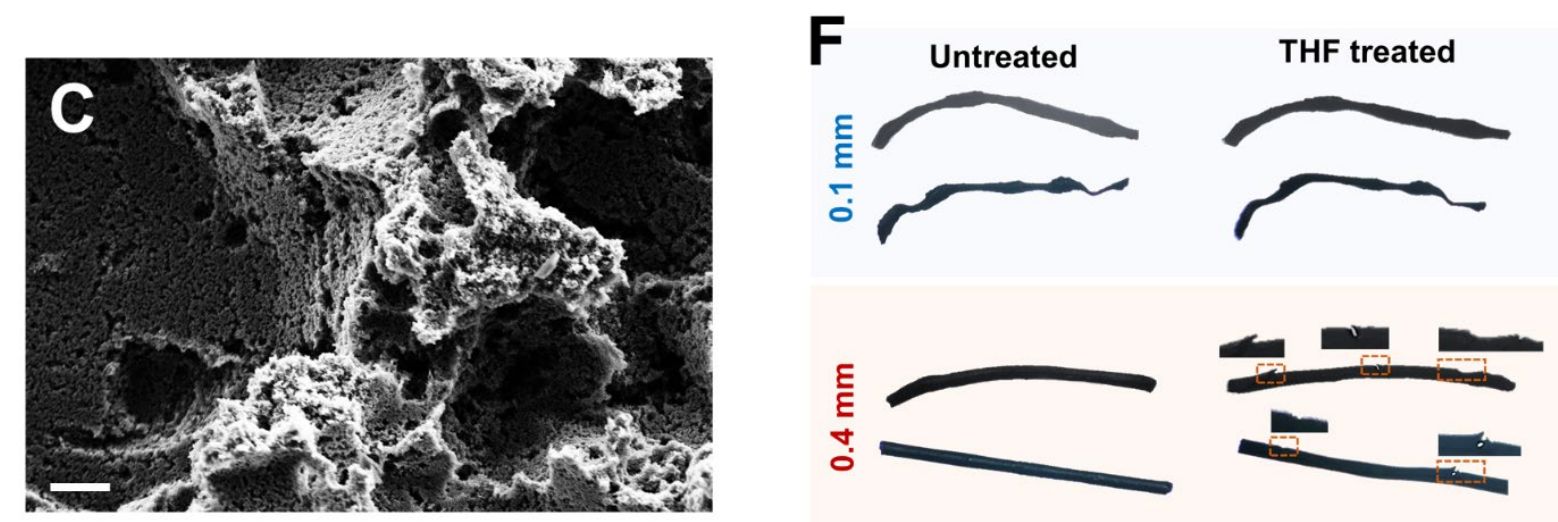

Figure 5. Investigation of the properties of the conductive carbon filament (A) Representative SEM image of THF treated filament showing the aggregation of CB particles. Scale bar is 20 
$\mu m$. (B) Representative SEM images of CB particles after dissolution of the filament in THF. Scale bar is $100 \mu \mathrm{m}$. (C) Zoomed in image of aggregated CB particles from the dissolved filament. Scale bar is $5 \mu \mathrm{m}$. (D) Mean particle size analysis of powdered $C B, n=8$. (E) \% loss in surface area for $0.1-0.4 \mathrm{~mm}$ layer thickness. (F) Two replicates of $0.1 \mathrm{~mm}$ and $0.4 \mathrm{~mm}$ pre and post THF treatment $(n=6-8)$ Insets show deformities in $0.4 \mathrm{~mm}$ after treatment. Data are shown as mean \pm S.D., $\mathrm{n}=6-8$. Data are shown as mean \pm S.D.

Based on all our findings, we hypothesise that 3D-printing at a vertical orientation at lower print layer heights results in electrodes with the fastest electron transfer kinetics and lowest charge transfer resistance. This is due to a combination of different reasons that are represented in Figure 6. Firstly, the air voids that form between filaments during the FFF printing process causes more resistance, where the size of these air voids are bigger in larger print layers. This increases resistivity as well as reduces the surface area of point-to-point contact which is critical for horizontal prints to have complete conductive pathways from electrical contact to solution interface. In vertical prints, conductive pathways can be achieved within individual print layers, which are all capable of doing singular connections from electrical contact to solution interface. Therefore, the effect of air voids is less problematic in vertical prints (Figure 6).

Second, due to the higher density of the CB particles in comparison to the PLA, it is probable that during the heated printing process, the denser CB will arrange themselves more towards the centre, pushing the less dense PLA to the sides. These benefit the vertical orientation as conductive pathways are parallel to the direction of electrical connection and formed in a more ordered and compact structure in the lower print thicknesses, as can be seen from the schematic in Figure 6. These compact particles at $0.1 \mathrm{~mm}$ print layer thickness will also have a higher probability of forming horizontal conductive pathways between the printed layers. On the other hand, in the 
$0.4 \mathrm{~mm}$ print layer thickness, there is scope for more random structuring of the CB particles, which will reduce the probability of forming successful pathways between the layers, or within the layer itself. Hence, a $0.4 \mathrm{~mm}$ layer will significantly less vertical conductive pathways formed in comparison to four $0.1 \mathrm{~mm}$ layers, which thus explains the facilitated electron transfer kinetics and improved interfacial charge-transfer resistance in the lower printing layers.
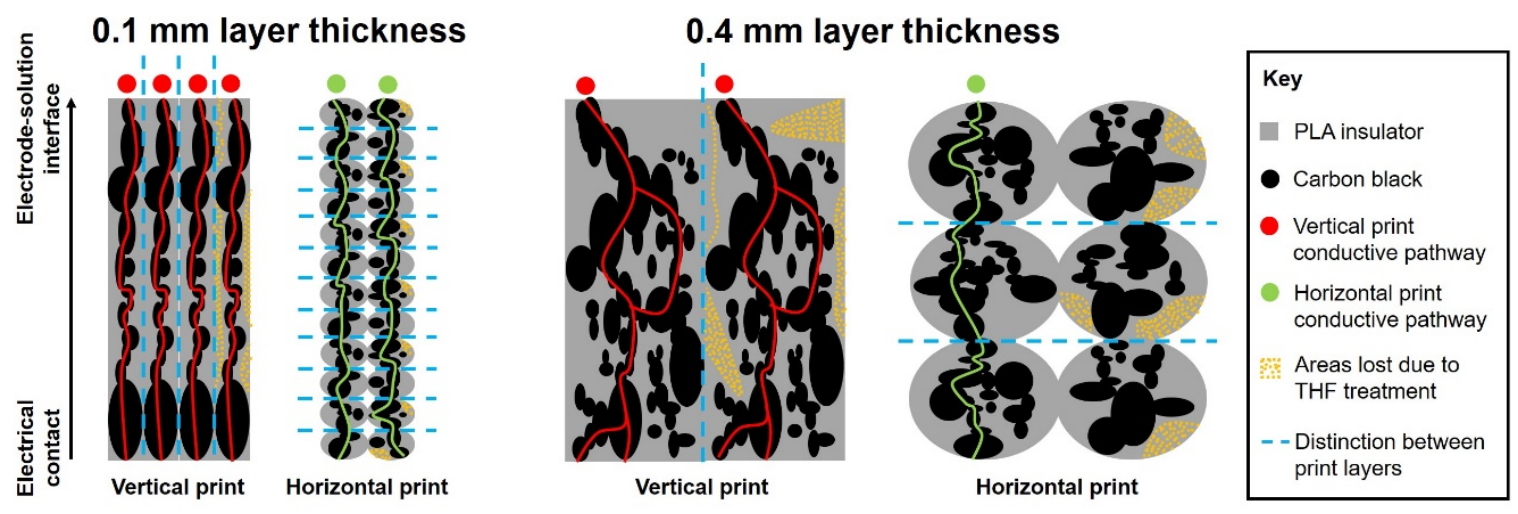

Figure 6. Schematic displaying the differences in the horizontal and vertical prints of 0.1 and $0.4 \mathrm{~mm}$ and the different conductive pathways formed in consecutive print layers.

\section{Conclusions}

We have shown that 3D-printing parameters can have a significant influence on the electron transfer kinetics of 3D-printed CB/PLA electrodes. Within our study, we have shown that with increasing print layer thickness, there is a reduction in conductivity of the material, which is further reduced if the print is in a horizontal rather than vertical orientation. Our study highlights the important influence that 3D printing parameters have on electron transfer kinetics and therefore provides key insight into the fundamental influence of such factors when designing and fabricating electrodes for electrochemical sensing and electronic applications. 


\section{Acknowledgements}

This work was funded by CRUK ESPRC Multidisciplinary Project Award (C57783/A24316; NS/A000065/1) and IMPRESSPlus EPSRC grant (EP/N027345/1). Authors would like to acknowledge Dr. Jon Salvage, Mareike Hermann, and Emma Ward for experimental assistance and Dr. Marcus Dymond for valuable theoretical discussions.

\section{Conflict of interest}

The authors declare no conflict of interest.

\section{References}

[1] M. Cheng, R. Deivanayagam, R. Shahbazian-Yassar, 3D Printing of Electrochemical Energy Storage Devices: A Review of Printing Techniques and Electrode/Electrolyte Architectures, Batteries \& Supercaps, 3 (2020) 130-146.

[2] A. Zhakeyev, P. Wang, L. Zhang, W. Shu, H. Wang, J. Xuan, Additive Manufacturing: Unlocking the Evolution of Energy Materials, Adv Sci (Weinh), 4 (2017) $1700187-1700187$

[3] U. Gulzar, C. Glynn, C. O’Dwyer, Additive manufacturing for energy storage: Methods, designs and materials selection for customizable 3D printed batteries and supercapacitors, Current Opinion in Electrochemistry, (2020). 
[4] A. Abdalla, B.A. Patel, 3D-printed electrochemical sensors: A new horizon for measurement of biomolecules, Current Opinion in Electrochemistry, 20 (2020) 78-81. [5] N. Rohaizad, C.C. Mayorga-Martinez, F. Novotný, R.D. Webster, M. Pumera, 3Dprinted $\mathrm{Ag} / \mathrm{AgCl}$ pseudo-reference electrodes, Electrochemistry Communications, 103 (2019) 104-108.

[6] V. Katseli, A. Economou, C. Kokkinos, Single-step fabrication of an integrated 3Dprinted device for electrochemical sensing applications, Electrochemistry Communications, 103 (2019) 100-103.

[7] M.P. Browne, M. Pumera, Impurities in graphene/PLA 3D-printing filaments dramatically influence the electrochemical properties of the devices, Chemical Communications, 55 (2019) 8374-8377.

[8] C.L. Manzanares Palenzuela, F. Novotný, P. Krupička, Z. Sofer, M. Pumera, 3DPrinted Graphene/Polylactic Acid Electrodes Promise High Sensitivity in Electroanalysis, Analytical Chemistry, 90 (2018) 5753-5757.

[9] M. Zafir Mohamad Nasir, F. Novotny, O. Alduhaish, M. Pumera, 3D-printed electrodes for the detection of mycotoxins in food, Electrochemistry Communications, (2020) 106735.

[10] V. Katic, P.L. dos Santos, M.F. dos Santos, B.M. Pires, H.C. Loureiro, A.P. Lima, J.C.M. Queiroz, R. Landers, R.A.A. Muñoz, J.A. Bonacin, 3D Printed Graphene Electrodes Modified with Prussian Blue: Emerging Electrochemical Sensing Platform for Peroxide Detection, ACS Applied Materials \& Interfaces, 11 (2019) 35068-35078. [11] M.P. Browne, F. Novotný, Z. Sofer, M. Pumera, 3D Printed Graphene Electrodes' Electrochemical Activation, ACS Applied Materials \& Interfaces, 10 (2018) 4029440301. 
[12] R. Gusmão, M.P. Browne, Z. Sofer, M. Pumera, The capacitance and electron transfer of 3D-printed graphene electrodes are dramatically influenced by the type of solvent used for pre-treatment, Electrochemistry Communications, 102 (2019) 83-88. [13] D.M. Wirth, M.J. Sheaff, J.V. Waldman, M.P. Symcox, H.D. Whitehead, J.D. Sharp, J.R. Doerfler, A.A. Lamar, G. LeBlanc, Electrolysis Activation of FusedFilament-Fabrication 3D-Printed Electrodes for Electrochemical and Spectroelectrochemical Analysis, Analytical Chemistry, 91 (2019) 5553-5557.

[14] M. Zafir Mohamad Nasir, F. Novotný, O. Alduhaish, M. Pumera, 3D-printed electrodes for the detection of mycotoxins in food, Electrochemistry Communications, $115(2020) 106735$.

[15] C. Kalinke, N.V. Neumsteir, G.d.O. Aparecido, T.V.d.B. Ferraz, P.L. dos Santos, B.C. Janegitz, J.A. Bonacin, Comparison of activation processes for 3D printed PLAgraphene electrodes: electrochemical properties and application for sensing of dopamine, Analyst, 145 (2020) 1207-1218.

[16] E.G. Gordeev, A.S. Galushko, V.P. Ananikov, Improvement of quality of 3D printed objects by elimination of microscopic structural defects in fused deposition modeling, PLoS One, 13 (2018) e0198370-e0198370.

[17] P. Chang, H. Mei, S. Zhou, K.G. Dassios, L. Cheng, 3D printed electrochemical energy storage devices, Journal of Materials Chemistry A, 7 (2019) 4230-4258.

[18] H.H. Bin Hamzah, O. Keattch, D. Covill, B.A. Patel, The effects of printing orientation on the electrochemical behaviour of $3 \mathrm{D}$ printed acrylonitrile butadiene styrene (ABS)/carbon black electrodes, Scientific Reports, 8 (2018) 9135.

[19] Ossila, Sheet Resistance: A Guide to Theory, 2020.

[20] R.S. Nicholson, Theory and Application of Cyclic Voltammetry for Measurement of Electrode Reaction Kinetics, Analytical Chemistry, 37 (1965) 1351-1355. 
[21] M.C. Bonetto, F.F. Muñoz, V.E. Diz, N.J. Sacco, E. Cortón, Fused and unzipped carbon nanotubes, electrochemically treated, for selective determination of dopamine and serotonin, Electrochimica Acta, 283 (2018) 338-348.

[22] R.L. McCreery, Advanced Carbon Electrode Materials for Molecular Electrochemistry, Chemical Reviews, 108 (2008) 2646-2687.

[23] R.S. Nicholson, I. Shain, Theory of Stationary Electrode Polarography. Single Scan and Cyclic Methods Applied to Reversible, Irreversible, and Kinetic Systems, Analytical Chemistry, 36 (1964) 706-723.

[24] E.P. Randviir, A cross examination of electron transfer rate constants for carbon screen-printed electrodes using Electrochemical Impedance Spectroscopy and cyclic voltammetry, Electrochimica Acta, 286 (2018) 179-186.

[25] I. Miccoli, F. Edler, H. Pfnür, C. Tegenkamp, The 100th anniversary of the fourpoint probe technique: the role of probe geometries in isotropic and anisotropic systems, Journal of Physics: Condensed Matter, 27 (2015) 223201.

[26] C. Bellehumeur, L. Li, Q. Sun, P. Gu, Modeling of Bond Formation Between Polymer Filaments in the Fused Deposition Modeling Process, Journal of Manufacturing Processes, 6 (2004) 170-178.

[27] S. Garzon-Hernandez, D. Garcia-Gonzalez, A. Jérusalem, A. Arias, Design of FDM 3D printed polymers: An experimental-modelling methodology for the prediction of mechanical properties, Materials \& Design, 188 (2020) 108414.

[28] S.A. Tronvoll, T. Welo, C.W. Elverum, The effects of voids on structural properties of fused deposition modelled parts: a probabilistic approach, The International Journal of Advanced Manufacturing Technology, 97 (2018) 3607-3618. 
[29] F. Rodriguez Jose, P. Thomas James, E. Renaud John, Characterization of the mesostructure of fused-deposition acrylonitrile-butadiene-styrene materials, Rapid Prototyping Journal, 6 (2000) 175-186.

[30] F. Rodríguez José, P. Thomas James, E. Renaud John, Mechanical behavior of acrylonitrile butadiene styrene (ABS) fused deposition materials. Experimental investigation, Rapid Prototyping Journal, 7 (2001) 148-158.

[31] E.P. Randviir, C.E. Banks, Electrochemical impedance spectroscopy: an overview of bioanalytical applications, Analytical Methods, 5 (2013) 1098-1115.

[32] S. Vogt, Q. Su, C. Gutiérrez-Sánchez, G. Nöll, Critical View on Electrochemical Impedance Spectroscopy Using the Ferri/Ferrocyanide Redox Couple at Gold Electrodes, Analytical Chemistry, 88 (2016) 4383-4390.

[33] S.D. Keighley, P. Estrela, P. Li, P. Migliorato, Optimization of label-free DNA detection with electrochemical impedance spectroscopy using PNA probes, Biosensors and Bioelectronics, 24 (2008) 906-911.

[34] H. Watschke, K. Hilbig, T. Vietor, Design and Characterization of Electrically Conductive Structures Additively Manufactured by Material Extrusion, Applied Sciences, 9 (2019) 779.

[35] S.W. Kwok, K.H.H. Goh, Z.D. Tan, S.T.M. Tan, W.W. Tjiu, J.Y. Soh, Z.J.G. Ng, Y.Z. Chan, H.K. Hui, K.E.J. Goh, Electrically conductive filament for 3D-printed circuits and sensors, Applied Materials Today, 9 (2017) 167-175.

[36] A. Grémare, V. Guduric, R. Bareille, V. Heroguez, S. Latour, N. L'heureux, J.-C. Fricain, S. Catros, D. Le Nihouannen, Characterization of printed PLA scaffolds for bone tissue engineering, Journal of Biomedical Materials Research Part A, 106 (2018) 887-894. 


\section{ToC Entry}

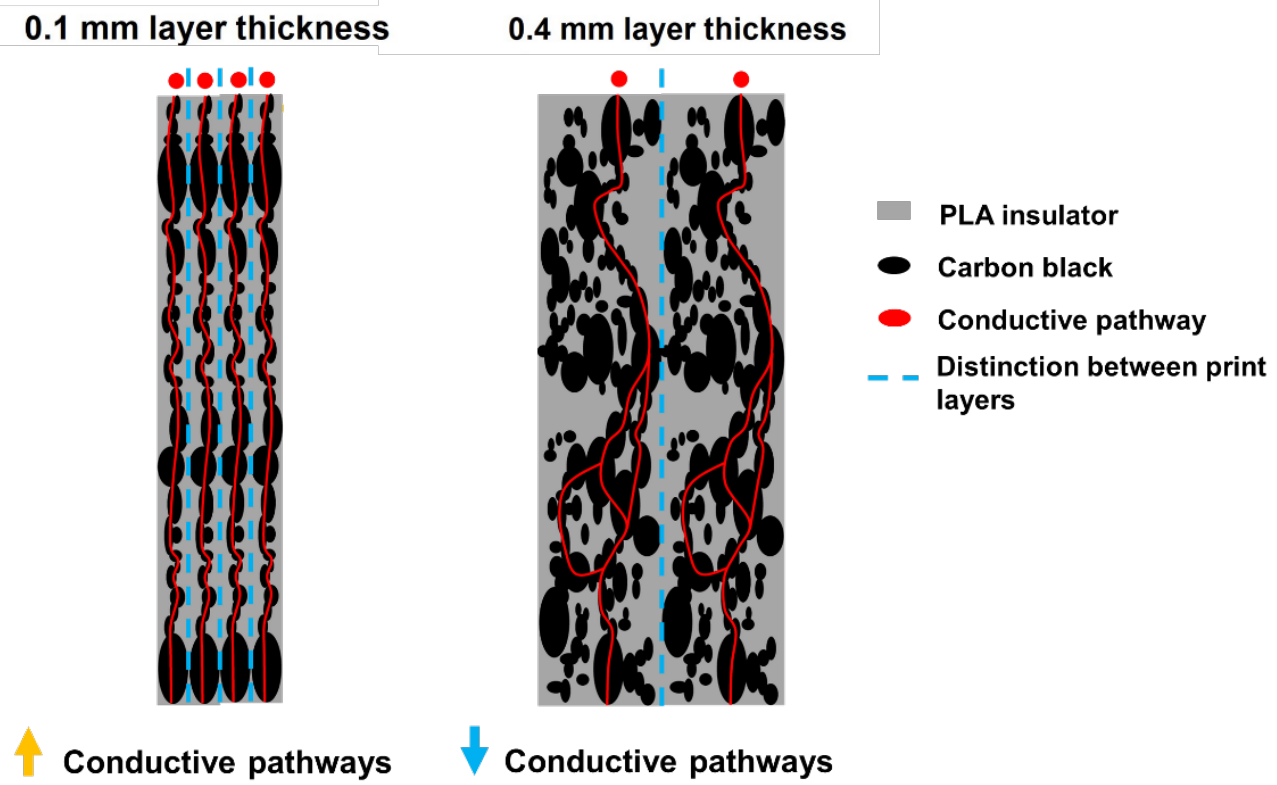

Electron transfer in 3D-printed electrodes: 3d-printed electrodes are printed in vertical and horizontal orientation with thickness ranging from $0.1-0.4 \mathrm{~mm}$. Electron transfer kinetics are enhanced in vertical orientation at lower print layer thickness due to the formation of less air voids and more conductive pathways. 